\title{
Editorial: The Value and Status of Replications in Animal Behavior and Cognition Research
}

\author{
Michael J. Beran \\ Department of Psychology, Georgia State University, Atlanta, GA \\ Citation - Beran, M. J. (2020). Editorial: The value and status of replications in animal behavior and cognition \\ research. Animal Behavior and Cognition, 7(1), i-iii. doi: https://doi.org/10.26451/abc.07.01.01.2020 \\ *Corresponding author (Email: mberan1@gsu.edu) \\ Abstract - This editorial discusses the need and value of replication efforts in comparative cognition and a call for \\ papers for a special issue on this topic. It highlights the contribution of Farrar et al. (2020, this issue) in helping \\ describe the problems and prospects for replication in comparative research.
}

Keywords - Editorial; Replication; Pre-registration; Animal cognition; Animal behavior

This issue leads with an important and timely article on replication efforts in comparative cognition (Farrar et al. 2020). As many readers will know, concerns about replicability in psychology (and other disciplines) have increased, and some have argued are even to the point of creating a "crisis" for some fields (Baker, 2016; Camerer et al., 2016, 2018; Open Science Collaboration, 2015; Shrout \& Rodgers, 2018; Stroebe \& Strack, 2014). This is an issue that affects those of us who work in comparative cognition, animal behavior, and comparative psychology. Farrar et al. outline the nature of the "replication crisis" and then specifically focus their efforts on describing their view of what can and perhaps cannot be done in comparative cognition. This is an important article, and one we hope will be widely read and disseminated among those who work to understand the behavior and cognition of nonhuman animals. This does not mean the editors of this journal endorse or agree with all points raised in Farrar et al., but we certainly see substantial merit and value in the views in this paper.

The need for replication is central to understanding better the nature of animal cognition and behavior, and it also aids in preventing some of the unintentional and perhaps intentional practices of selectively reporting data (such as " $p$-hacking"; Stevens, 2017). My own thoughts on this topic have been outlined elsewhere (Beran, 2018), but I want to highlight a few issues. First, the opportunity for preregistration of methods and analysis plans is an important tool for comparative researchers moving forward, as is true in many areas of science. What is perhaps most ideal is the approach in which preregistrations are peer-reviewed for scientific rigor in terms of proposed methodology and data analysis, and theoretical interest, before any data are collected (Nosek \& Lakens, 2014). This has the advantage of increasing our confidence in the resulting data as reflecting accurately the nature of animal behavior and cognition. But, equally important (in my opinion) is that this approach allows investigators to confirm with outside experts that their hypotheses, ways to address those hypotheses, and manner of analyzing the resulting data are all valid (and valuable), and this is all done before any biases emerge based on the results. The practical outcome of this is a commitment by publishers that those data "see the light of day" and are not subject to the file-drawer problem (null results that are never published) or the "Reviewer 2 problem" (as I call it) where dislike of an outcome because of its implications leads to difficulty in getting 
those data published. Peer reviewer pre-registration is a particularly valuable tool for those laboratories that want to replicate a controversial or high-visibility result because agreement that the replication is solid protects from subsequent efforts to block publication of data that may not support the narrative that led to the replication effort.

For these reasons, and others such as those raised by Farrar et al. (2020) and Stevens (2017), Animal Behavior and Cognition was among the first journals in comparative behavioral and cognitive research to offer pre-registered reports (Vonk \& Krause, 2018). However, we have received very few submissions of this type, and so I wanted to take this opportunity to remind the readers of this journal that there is this opportunity. The process is not complicated, and yet it offers the assurance that a welldesigned study will have the resulting data shared with the scientific community. We recognize (and agree) that there are many projects one would conduct in comparative psychology and animal behavior that do not lend themselves to pre-registration. We will still need to do exploratory experiments, and then modify or adjust methods based on preliminary results, or even build hypotheses that emerge from early results. However, the pre-registered format is ideal when there are clear hypotheses, well-described methods, and planned analyses of data. And, this is true for new studies and new research topics as well as for potential replications.

Animal Behavior and Cognition also currently has an open invitation for submissions to a future special issue titled "The Value and Status of Replications in Animal Behavior Research." Here is the call:

Replications are widely considered an essential tool to evaluate scientific claims. Recent efforts in psychology and related fields have highlighted the poor replicability of many research findings and well-known effects and question the lack of incentives for researchers to replicate former studies. These efforts have led to a large-scale movement within psychology with the aim to both study and address these issues. A similar development has not happened in animal behavior science, although related concepts and issues are being considered and discussed by different researchers. To provide a more organized and coherent framework for discussions on this topic as well as bring together researchers and thoughts from different disciplines related to animal behavior, we are organizing a special issue of Animal Behavior and Cognition (anticipated publication in May 2021). Submissions can be reviews, historical perspectives, commentaries, simulation studies and empirical papers. Regarding the latter, the special issue also offers the opportunity to publish close replication studies - we thus especially want to emphasize submission of replication attempts that did or did not confirm the original results.

We believe this special issue will build on the important contributions made in Farrar et al. (2020) and Stevens (2017). We also would be pleased to receive commentaries on the Farrar et al. paper for that special issue of Animal Behavior and Cognition. If you have any questions, please email animalbehaviorandcognition@gmail.com. We can provide more details and put you in touch with the guest editors of that special issue.

\section{References}

Baker, M. (2016). 1,500 scientists lift the lid on reproducibility. Nature News, 533, 452-454.

Camerer, C. F., Dreber, A., Forsell, E., Ho, T.-H., Huber, J., Johannesson, M., ... Wu, H. (2016). Evaluating replicability of laboratory experiments in economics. Science, 351, 1433-1436.

Camerer, C. F., Dreber, A., Holzmeister, F., Ho, T.-H., Huber, J., Johannesson, M., ... Wu, H. (2018). Evaluating the replicability of social science experiments in Nature and Science between 2010 and 2015. Nature Human Behaviour, 2, 637-644.

Farrar, B. G., Boeckle, M., \& Clayton, N. S. (2020). Replications in comparative cognition: What should we expect and how can we improve? Animal Behavior and Cognition, 7, 1-22.

Nosek, B. A., \& Lakens, D. D. (2014). Registered reports: A method to increase the credibility of published results. Social Psychology, 45, 137-141. 
Open Science Collaboration (2015). Estimating the reproducibility of psychological science. Science, 349 , aac4716. Shrout, P. E., \& Rodgers, J. L. (2018). Psychology, science, and knowledge construction: Broadening perspectives from the replication crisis. Annual Review of Psychology, 69, 487-510.

Stevens, J. R. (2017). Replicability and reproducibility in comparative psychology. Frontiers in Psychology, $8,862$.

Stroebe, W., \& Strack, F. (2014). The alleged crisis and the illusion of exact replication. Perspectives on Psychological Science, 9, 59-71.

Vonk, J. \& Krause, M. A. (2018). Editorial: Announcing preregistered reports. Animal Behavior and Cognition, 5, iii. 